\title{
Metodología para la priorización de tecnologías emergentes de recobro mejorado químico
}

\author{
Claudia Lorena Delgadillo Aya ${ }^{1 *}$; Venus Minerva Díaz Guardia ${ }^{2}$; Gustavo Adolfo Maya Toro; \\ Rubén Hernán Castro García ${ }^{1}$ y Henderson Ivan Quintero Pérez ${ }^{1}$. \\ ${ }^{1}$ Instituto Colombiano del Petróleo (ICP). Vía Piedecuesta Km 7. Piedecuesta, Colombia \\ ${ }^{2}$ Universidad industrial de Santander (UIS). Carrera 27 Calle 9. Bucaramanga, Colombia \\ E-mail: claudia.delgadillo@ecopetrol.com.co
}

\begin{abstract}
Resumen
Debido al incremento en el consumo de combustibles y los retos que representa la reposición de reservas, la implementación de procesos de recobro mejorado juega un papel fundamental en el balance energético mundial, ya sea mediante el incremento del factor de recobro o la incorporación de reservas. Mejorar el desempeño de los procesos de recobro mejorado es una necesidad de la industria petrolera actual y por ello las investigaciones relacionadas con esta área de conocimiento crecen constantemente. Por esta razón, en el desarrollo de este trabajo se ha diseñado una metodología que permite priorizar tecnologías emergentes de recobro mejorado que permitan identificar aquellas con un mayor potencial de implementación en campo a mediano plazo ajustada a las necesidades de los yacimientos colombianos. La metodología desarrollada se puede aplicar a priorización de tecnologías en general, sin embargo, el enfoque inicial se centró en identificar tecnologías emergentes de recobro químico que incluyen: inyección de polímeros modificados y con diferentes funcionalidades, nanotecnología para recobro mejorado, nuevos surfactantes, inyección de agua modificada y disociaciones fuertes de sustancias químicas. Teniendo en cuenta lo novedoso de las tecnologías y lo embrionario de su simulación física y numérica, se definieron parámetros que permitirían su valoración en términos de viabilidad de aplicación: grado de madurez del proceso utilizando el concepto de Technology Readiness Levels (TRL), eficiencia de recobro esperada, reservas disponibles empleando el concepto de analogías de Security Energy Commission (SEC), costos asociados, comercialidad, alineación estratégica con las necesidades de la industria petrolera colombiana y por último, el potencial impacto ambiental. Finalmente, a partir de la definición de los parámetros de screening técnico para cada tecnología y la evaluación de los parámetros valorativos de la metodología, se realizó un proceso comparativo de la potencialidad de las tecnologías con las necesidades de los campos colombianos, obteniendo un ranking preliminar de tecnologías aplicables y donde la inyección de polímeros modificados y la inyección de agua modificada resultaron ser las tecnologías de mayor potencial de acuerdo a los criterios desarrollados en el presente trabajo. La priorización de estas tecnologías permite concentrar esfuerzos en la selección de aquellas con mayor potencial con el objetivo de materializar planes de producción en el mediano plazo, así como apalancar la incorporación de reservas.
\end{abstract}

Palabras clave: Tecnologías emergentes, recobro químico, screening, priorización de tecnologías, polímeros modificados, agua modificada.

\section{Methodology to rank emerging technologies of chemical enhanced oil recovery methods}

\begin{abstract}
Due to the increase in energy consumption and current challenges replacing reserves, the implementation of enhanced oil recovery (EOR) processes will continue playing a fundamental role in the global energy balance, either by increasing the recovery factor or by incorporating reserves. Therefore, increase the efficiency of EOR methods represents an important challenge in today's oil industry and its research is showing a growing interest. For this reason, this work developed a methodology to rank emerging technologies of chemical EOR (CEOR) that can be implemented in Colombian fields in the mid-term. The proposed methodology can be implemented for the prioritization of technologies in general. However, this work is focused on identifying CEOR emerging technologies such as: modified polymers with different functionalities, nanotechnology for CEOR, new surfactants, modified water injection and strong dissociations of chemical substances. Taking into account the novelty of these technologies and the limited number of protocols available to properly evaluate it at laboratory and numerical scale, a set of parameters to assess these technologies in terms of viability for field implementation were defined. These parameters include:
\end{abstract}

Cita: Delgadillo Aya, C. L., Díaz Guardia, V. M., Maya Toro, G. A., Castro García R. H. y Quintero Pérez, H. I. (2018). Metodología para la priorización de tecnologías emergentes de recobro mejorado químico. Revista Fuentes: El reventón energético, 16(2), 41-53. 
process maturity degree using Technology Readiness Levels (TRL) concept, expected recovery efficiency, available reserves and Security Energy Commission (SEC) reservoir analogy, associated costs, commercialization, strategic alignment with the Colombian oil industry needs and, finally, the potential environmental impact. Based on the definition of technical screening parameters for each technology and the evaluation of the set of parameters defined in the proposed methodology, a comparative process of the potential of each of the technologies evaluated with the needs of the Colombian fields was carried out. The results of this ranking methodology identified the injection of modified polymer with functionalities and the injection of modified water as the technologies with the largest potential for field implementation. Finally, the ranking of these CEOR technologies will allow focusing efforts in the selection of those with greater potential optimizing time and resources. In addition, it will contribute materializing field development plans, extending production life of Colombian assets as well as supporting the incorporation of oil reserves in the country.

Keywords: Emerging technologies, chemical enhanced oil recovery, screening, technology prioritization, modified polymers, modified water.

\section{Introducción}

Los métodos de recobro que se conocen y aplican de forma convencional como la inyección de agua, polímeros y vapor presentan cada vez mayores desafíos de implementación debido a condiciones de yacimiento retadoras para sus rangos de aplicación. Como Molano, Navarro y Díaz (2014) señalan, todos los procesos de recobro, convencionales o no, requieren esfuerzos de planeación, diseño, análisis experimentales y simulación numérica, entre otros.

En resumen esfuerzos investigativos y económicos importantes que hacen que no sea viable la implementación de todas las tecnologías existentes. Por lo tanto, es necesario valorar las tecnologías emergentes con mejor competencia financiera, que muestren incrementos en la eficiencia de recobro con mayores márgenes de aplicabilidad y en lo posible menos sujetos a las variaciones del precio del crudo.

Castro, Maya, Jimenez, Quintero, Díaz, Colmenares \& Perez (2016) refieren que en Colombia se han desarrollado proyectos con miras a lograr el entendimiento, apropiación, desarrollo e implementación de diferentes tecnologías, incluyendo procesos de conformance, inyección de geles para control de movilidad e inyección de polímeros. Sin embargo, la complejidad de algunos de los yacimientos del país impacta los indicadores financieros de una posible expansión de métodos convencionales de recobro mejorado.

Las tecnologías de recobro químico se presentan con la posibilidad de ampliar los rangos de aplicación de los métodos de recobro convencionales y su aplicación podría ejecutarse en el mediano plazo. En este sentido, para permitir la evaluación de la viabilidad de implementación de procesos de recobro mejorado es necesario realizar análisis de screening y analogías, basados en el estudio de casos implementados en campo ya sean exitosos o no, utilizando herramientas (software) específicos para el desarrollo de este tipo de evaluaciones (Delgadillo, Trujillo, Palma, Niz, Rodríguez \& Leon, 2014). Sin embargo, para el caso de las tecnologías emergentes, cuyo grado de desarrollo es bajo, no se pueden seleccionar de la misma forma.

Por lo anterior, en el desarrollo de este trabajo se propone una metodología que permita priorizar estas tecnologías, para enfilar los esfuerzos investigativos en aquellas que pueden generar mayor potencial de recobro incremental; aplicándola, en este caso, a las tecnologías emergentes de recobro químico y su posible aplicación a los campos colombianos, todo con el objetivo de establecer una jerarquización de tecnologías con potencial de aplicación y que respondan a las necesidades de los campos del país.

\section{Metodología}

La necesidad de expandir el rango de operación de proyectos de recobro mejorado hace que las tecnologías emergentes de recobro químico se presenten con un potencial de mejoramiento de la eficiencia de recobro de los campos petroleros, ya que ofrecen posibles soluciones tecnológicas al desarrollo convencional de métodos de recobro mejorado. Para el caso de los campos colombianos es necesario priorizar las tecnologías emergentes dependiendo de su potencial de aplicación y teniendo en cuenta las necesidades de los campos de interés; por lo anterior, se propone una metodología que permite priorizar estas tecnologías con base a criterios clave, para de esta forma enfocar los esfuerzos en aquellas de mayor aplicabilidad.

Una revisión generalizada de metodologías para selección de tecnologías permite identificar como requisitos básicos las dimensiones de economía, tecnología y sostenibilidad (Choudry, Muller, Alber, Riedel \& Landgrebe, 2018); otros autores, en función 
del área de conocimiento en que se desarrolle el estudio involucrarán un conjunto de nuevos criterios alineados a sus necesidades, pero generalmente el enfoque es económico; también se encuentran metodologías relacionadas con la priorización de campos sujetos a posible aplicación de métodos convencionales de recobro (Reyes, Trujillo, Velasquez \& Aya, 2017). Sin embargo, para el caso de la priorización de tecnologías emergentes de recobro químico es difícil la asignación o confiabilidad de costos e ingresos, ya que en su mayoría se encuentran en estado embrionario y sin experiencias de casos implementados a escala de campo. Por lo tanto, se presenta una metodología que de forma rápida permite evaluar y preseleccionar tecnologías de recobro químico, basado en criterios factibles de evaluar a la luz de su desarrollo técnico actual.

En la figura 1 se presenta la metodología diseñada de forma esquemática y posteriormente se describe cada uno de sus componentes.

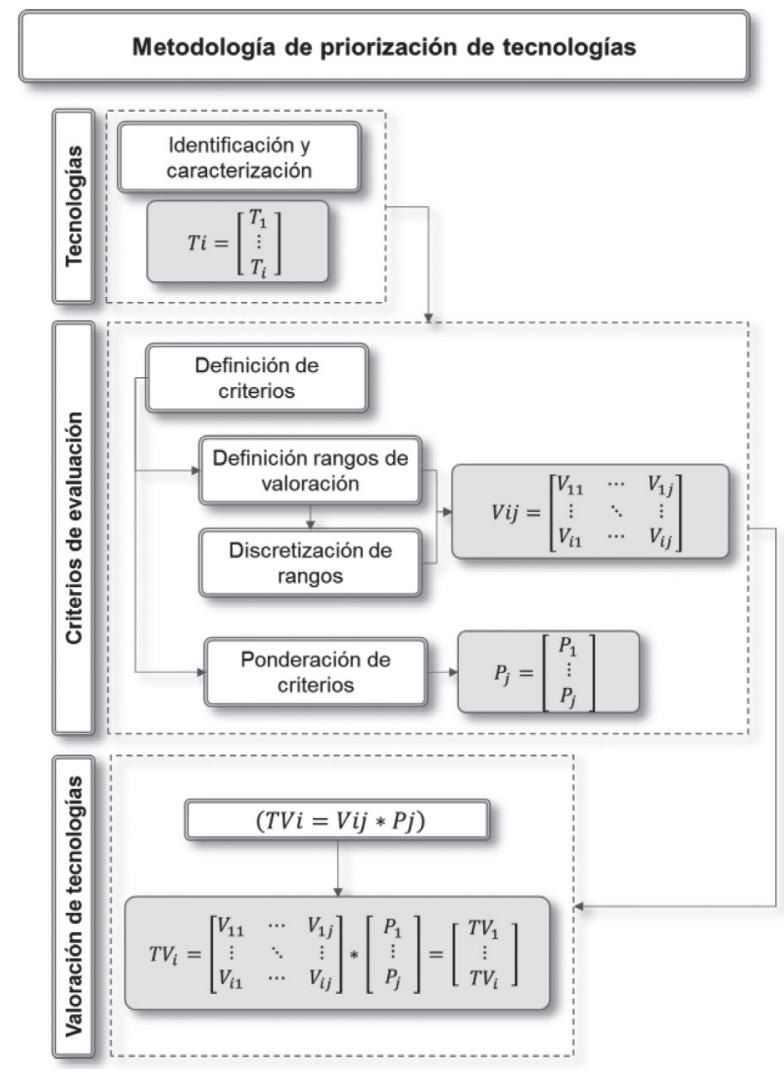

Figura 1. Esquema de la metodología para la priorización de tecnologías emergentes.

\section{Identificación de tecnologías}

Como primer paso de la metodología propuesta se requiere generar un listado de tecnologías potenciales de aplicación, las cuales serán identificadas por un equipo interdisciplinario que garantice que se evaluarán todas aquellas tecnologías disponibles, que puedan brindar una solución a las necesidades de los activos y que a la vez se encuentren alineadas con su estrategia de desarrollo. Para esta tarea se recomienda realizar talleres de lluvia de ideas, ejercicios de vigilancia tecnológica y alinear con los mapas de ruta tecnológica de la compañía.

Luego de identificar las tecnologías es necesario realizar una caracterización de las mismas, lo que permite establecer los rangos de operación y plantear el posterior análisis, cuanto mayor conocimiento se tenga, la valoración será realizada de forma más precisa y la metodología se implementará de manera más eficiente. En este sentido, a continuación se presenta una descripción de las tecnologías preseleccionadas para el caso de las tecnologías emergentes de recobro químico.

\section{Inyección de polímeros modificados y con funcionalidades}

Desde hace varias décadas los polímeros utilizados comúnmente en procesos de recobro mejorado de hidrocarburos (EOR, Enhanced Oil Recovery) son poliacrilamidas parcialmente hidrolizadas (HPAM, Partially Hydrolyzed Polyacrylamide) y sus derivados, así lo refieren Albonico \& Lockhart (1993) y Seright (2016); sin embargo, estos presentan limitaciones en su rendimiento a condiciones de alta temperatura $\mathrm{y} / \mathrm{o}$ alta salinidad (HTHS, high temperature and high salinity) lo que puede llevar a una disminución significativa de la viscosidad (Wu, Mahmoudkhani, \& Watson, 2012), así como efectos adversos en la viscoelasticidad del fluido inyectado (Tapias, Lizcano, Moreno \& Zanoni, 2018). Por esto, en los últimos años gran parte de la investigación en el área se ha centrado en el desarrollo de nuevos polímeros con alto rendimiento ante condiciones HTHS, como lo refiere Thomas (2008). Un nuevo polímero con base en poliacrilamidas puede sintetizarse con el objeto de mejorar su rendimiento, los mecanismos mostrados por Xingcai \& Xion (2015) incluyen: aumento de la rigidez molecular, introducción de grupos laterales, introducción de un grupo tolerante a la sal, introducción de un grupo resistente a la hidrólisis, introducción de grupos que pueden inhibir la hidrólisis de amida e introducción de grupos hidrófobos.

\section{Nanotecnología para recobro mejorado}

En las últimas décadas la nanotecnología ha permitido el desarrollo de herramientas que tienen el potencial de incrementar la eficiencia de los procesos de 
exploración y producción de hidrocarburos, generando materiales con propiedades de alta calidad y amigables con el entorno de aplicación. De esta forma, la combinación de técnicas tradicionales de producción y aprovechamiento de hidrocarburos con aquellas provistas por la nanotecnología, redundará en un incremento notable en los volúmenes de petróleo recuperados con márgenes de ganancia aceptables en el mercado energético mundial. Khavkin (2014) ha presentado la posibilidad de incorporar nanotecnología en el recobro mejorado de hidrocarburos; en este sentido, los estudios más avanzados al momento se concentran en mejorar las propiedades de polímeros y surfactantes para recobro químico de hidrocarburos, así como, en utilizar nano catalizadores en procesos que incluyen mejora de crudo pesado (Ragab, 2014). Actualmente, entre las diferentes opciones de nanotecnología más utilizadas en EOR se encuentran las nanopartículas de sustancias afines a los yacimientos, tales como nano esferas de sílice. Sin embargo, autores como Ogolo, Olafuyi \& Onyekonwu (2012) y Nazari Moghaddam, Bahramian, Fakhroueian, Karimi \& Arya (2015), reportan el uso de nano partículas (NPs) de óxidos de metales tales como aluminio, zinc, magnesio, hierro, zirconio, níquel, estaño, titanio, cerio, cobre, silicio, y carbonato de calcio, evidenciando que la eficiencia de recobro se ve afectada por el tipo de óxido y el fluido en el cual este se dispersa. La evaluación de esta tecnología se realizará basados en procesos de recobro mejorado químico (CEOR) funcionalizados con nano partículas.

\section{Nuevos surfactantes}

Los smart surfactants o surfactantes mejorados surgen como una alternativa para mejorar el recobro con la asociación de dos o más mecanismos EOR, una de las técnicas que más sobresale en términos de costos y uso de tecnología convencional para recobro mejorado, es el uso de surfactantes poliméricos, ya que ofrece la posibilidad de combinar los efectos positivos de los polímeros y surfactantes en un solo componente. El potencial de estas sustancias para procesos EOR ha sido reportado por Winnik \& Yekta (1997) y por Elraies, Tan, Fathaddin, \& Abo-Jabal (2011), entre otros autores. La esencia de los surfactantes poliméricos se basa en la introducción de grupos hidrofóbicos en un polímero soluble en agua, muchas de estas estructuras emplean la poliacrilamida modificada hidrófobamente (HMPAM, hydrophobically modified polyacrylamides) o los polisacáridos (Wever, Picchioni \& Broekhuis, 2011), éstos grupos hidrofóbicos se incluyen principalmente para alterar la reología del sistema; la parte hidrófoba puede formar una asociación transitoria dependiente del cizallamiento en agua con posterior aumento de la viscosidad (Kudaibergenov \& Ciferri, 2007). Los estudios sobre las propiedades de los surfactantes poliméricos se centran principalmente en el efecto de las interacciones hidrofóbicas (Wever, Picchioni \& Broekhuis, 2014) o de la estructura química en la reología (Wever, Polgar, Stuart, Picchioni \& Broekhuis, 2013) mientras que son escasos los estudios que evalúan el efecto de la inclusión de la parte hidrófoba sobre las propiedades superficiales para procesos EOR.

\section{Inyección de agua modificada}

Está tecnología se enfoca en la mejora del proceso de inyección de agua convencional en busca de mejorar aspectos como la eficiencia de barrido, la eficiencia de desplazamiento y la aplicación a yacimientos que tradicionalmente no pueden ser sometidos a este tipo de proceso. La mejora sobre la inyección de agua convencional se logra mediante un incremento en la eficiencia de barrido microscópica. Gracias al trabajo de Jadhunandan \& Morrow (1995) el escenario más común de inyección de agua modificada corresponde a la inyección de agua de baja salinidad; adicionalmente, trabajos como los de Tang \& Morrow (1997 y 1999) reportan pruebas en campo que confirman producción incremental de esta tecnología, no obstante, no hay un consenso respecto a los fenómenos que causan este efecto y los mecanismos responsables para explicar los recobros incrementales. Los principales mecanismos que se reportan en la literatura incluyen la alteración de la mojabilidad de la roca, relacionado directamente con la capacidad de intercambio catiónico presente. La evaluación de esta tecnología en el presente trabajo incluye agua modificada ya sea de baja salinidad o un diseño de composición química del agua característico para el yacimiento de interés.

\section{Disociaciones fuertes de sustancias químicas}

Mediante un efecto combinado de procesos térmicos, químicos y de empuje de gas, esta tecnología promete beneficios que pueden ser implementados en proyectos de recobro químico, recobro post- co producción de crudo y arena (CHOPS, Cold Heavy Oil Production with Sand) y mejoramiento de movilidad en carbonatos. Su nombre técnico es Sodium Sílice Powder (NaSi) in Diesel. El fundamento de la tecnología se resume en la ecuación 1, en ella se observa que el siliciuro de sodio reaccionan con el agua produciendo hidrógeno, silicatos de sodio y calor (Krumrine, Lefenfeld \& Romney, 2016). 
$\mathrm{Na}_{4} \mathrm{Si}_{4}(s)+10 \mathrm{H}_{2} \mathrm{O}(l) \rightarrow 10 \mathrm{H}_{2}(g)+$

$2 \mathrm{Na}_{2} \mathrm{Si}_{2} \mathrm{O}_{5}(\mathrm{ag})+1654(\mathrm{kj})$

Esta reacción exotérmica ocurre en el yacimiento generando varios efectos sobre los fluidos in-situ que favorecen el incremento de la producción de aceite, tales como: reducción de viscosidad efecto del incremento de la temperatura $(1654 \mathrm{~kJ})$, aumento de la presión y potencial efecto de empuje por la formación de Hidrógeno, y formación de surfactantes naturales debido a la interacción con silicato de sodio generado durante la reacción. Se propone que la combinación de estos mecanismos contribuiría a la generación de incrementales de producción de petróleo. A la fecha, existen pocas aplicaciones de campo en yacimientos en Canadá desarrollados por CHOPS. Sin embargo, estas experiencias de campo no han sido documentadas en la literatura y es de aclarar que no se encuentran reportes en la literatura acerca de resultados de aplicación en campo de esta tecnología desde un enfoque CEOR.

\section{Inyección de bacterias (MEOR)}

La inyección de microorganismos con objeto de incrementar el factor de recobro de campos petroleros no es realmente una tecnología emergente, se ha reportado en la literatura desde 1946 como lo refieren Maudgalya, Knapp \& McInerney (2007); sin embargo, no es considerada aún una tecnología madura debido a la complejidad de sus procesos, los múltiples efectos potenciales y fenómenos envueltos, así como los pocos casos de aplicación en campo. El proceso envuelve tres elementos principales: los microbios, los nutrientes necesarios para su supervivencia y los bio catalizadores que aceleran la actividad bacteriana, (Portwood, 1995).

Los principales beneficios reportados en literatura producto de la implementación de la tecnología MEOR incluyen: estimulación y limpieza de pozos, modificación de perfiles de permeabilidad y control de movilidad, reducción de viscosidad por medio de biodegradación y gas o químicos (p.e. Biosurfactantes) generados por los microbios in situ. La investigación al respecto es extensa, no obstante, se considera un proceso altamente complejo y los resultados de pruebas en campo en procesos EOR van de ligeramente buenos a poco prometedores, (Gray, Yeung, Foght, \& Yarranton, 2008).

Para el desarrollo de la metodología y con base en las tecnologías identificadas, en adelante, el listado de tecnologías será reconocido como un vector $\mathrm{Ti}$, donde i representa cada una de las tecnologías a evaluar. En el caso de estudio, se identificó un conjunto de tecnologías de recobro químico que un equipo multidisciplinario estableció que cuentan con potencial de aplicación a mediano plazo. Por lo tanto, el vector Ti para el caso de estudio se define de acuerdo a la ecuación 2.

$$
T i=\left[\begin{array}{c}
\text { Nanotecnología para EOR } \\
\text { Agua modificada } \\
\text { Polímeros modificados } \\
\text { Nuevos surfactantes } \\
\text { Disociaciones de sustancias químicas } \\
\text { MEOR }
\end{array}\right]
$$

\section{Definición de criterios de evaluación}

Paralelo a la caracterización de las tecnologías, se deben definir los criterios a evaluar, los cuales conforman la matriz $\mathrm{Cjk}$, en donde $\mathrm{j}$ representa los diferentes criterios y $\mathrm{k}$ los rangos de valoración que pueden tomar. Para el caso de estudio, la priorización de tecnologías emergentes de recobro químico, se definieron siete criterios que evalúan en conjunto el nivel de madurez e incertidumbre de la tecnología, su impacto en reservas y económico, impacto ambiental, comercialidad y alineación estratégica del plan de negocios de la empresa. Cada criterio y su propuesta de valoración se resumen a continuación:

\section{Nivel de madurez de la tecnología}

Busca brindar una medida de la evolución de una tecnología, desde su nacimiento como idea hasta alcanzar un nivel comercial, brindando una dimensión del grado de incertidumbre en el éxito de la tecnología, a la vez que mide el nivel de innovación de la misma. Como referente para la medición de este criterio se toma la metodología Technology Readiness Levels (TRL), concebida originalmente por la NASA en el año 1974, y posteriormente incorporada al departamento de defensa de los Estados Unidos (U.S. DoD), la agencia espacial Europea (ESA) y la comisión europea (EC), entre otras grandes instituciones a nivel mundial (J. Banke, 2010). En la actualidad, el TRL considera 9 niveles que se extienden desde los principios básicos de la nueva tecnología hasta llegar a sus pruebas con éxito en un entorno operativo y comercial. La descripción de cada nivel o calificación se presenta en la tabla 1. 
Tabla 1. Technology Readiness Level.

\begin{tabular}{|c|c|}
\hline Nivel & Definición \\
\hline TRL 1 & Principios básicos observados. \\
\hline TRL 2 & Concepto de tecnología formulado. \\
\hline TRL 3 & Prueba experimental de concepto. \\
\hline TRL 4 & $\begin{array}{c}\text { Validación de componentes/subsistemas en pruebas de } \\
\text { laboratorio. }\end{array}$ \\
\hline TRL 5 & $\begin{array}{c}\text { Validación de sistema/subsistema/o componente en un } \\
\text { ambiente relevante. }\end{array}$ \\
\hline TRL 6 & $\begin{array}{c}\text { Demostración/validación de sistema, subsistema, modelo } \\
\text { o prototipo en un ambiente con condiciones relevantes } \\
\text { cercanas a las condiciones reales/operativas. }\end{array}$ \\
\hline TRL 7 & $\begin{array}{c}\text { Sistema/prototipo completo demostrado en ambiente } \\
\text { operacional. }\end{array}$ \\
\hline TRL 8 & $\begin{array}{c}\text { Sistema completo y calificado a través de pruebas y } \\
\text { demostraciones en ambientes operacionales. }\end{array}$ \\
\hline TRL 9 & Sistema operativo. \\
\hline
\end{tabular}

De acuerdo con la clasificación presentada en la tabla 1, cuando una tecnología está en TRL 1, la investigación científica está comenzando y esos resultados se están traduciendo en investigación y desarrollo futuros. TRL 2 ocurre una vez que los principios básicos han sido estudiados y las aplicaciones prácticas se pueden aplicar a esos hallazgos iniciales, entonces, la tecnología TRL 2 es especulativa, ya que hay poca o ninguna prueba experimental de concepto para la tecnología.

Cuando comienza la investigación y el diseño activo, una tecnología se eleva a TRL 3; en general, se requieren estudios analíticos y de laboratorio a este nivel para ver si una tecnología es viable y está lista para avanzar en el proceso de desarrollo. A menudo, durante TRL 3, se construye una prueba experimental o un modelo de prueba de concepto. Una vez que la prueba de concepto está lista, la tecnología avanza a TRL 4, durante el cual, las piezas de múltiples componentes se prueban entre sí, evaluando que funcionan conjuntamente en un sistema.

En TRL 5, la tecnología es sometida a pruebas rigurosas en un ambiente relevante, es decir entornos que sean lo más cercano al caso realista. En TRL 6 se tiene un prototipo completamente funcional o un modelo de representación. La tecnología TRL 7 requiere que el modelo de trabajo o prototipo se demuestre en un ambiente operacional. En TRL 8 ha sido probada y está lista para su implementación en un sistema tecnológico o de tecnología ya existente y una vez que una tecnología sido probada durante una aplicación exitosa, se considera que está disponible para su producción y/o comercialización, entonces alcanza el TRL 9.
Eficiencia de recobro. Los métodos de recobro generalmente se evalúan en términos de su factor de recobro, entendiéndose este como un porcentaje adicional de producción generado por la aplicación del método. Sin embargo, las tecnologías emergentes no tienen aplicaciones a escala de campo. Por lo tanto, no es posible asignar un factor de recobro asociado a su aplicación, pero si es posible establecer la eficiencia de recobro que se espera. En este sentido, el aceite que puede recuperarse con el uso de cualquier método de recobro, expresado como porcentaje del aceite total al inicio del proceso, puede

entenderse como la eficiencia global del desplazamiento y expresarse como le producto de una eficiencia microscópica $\left(E_{m}\right)$ por una eficiencia volumétrica $\left(E_{V}\right)$ y de acuerdo a la ecuación 3 :

$$
E=E_{m} E_{V}
$$

La eficiencia microscópica corresponde al porcentaje de aceite que el fluido desplazante logra movilizar en aquellos puntos del yacimiento donde hacen contacto, este es un proceso que tiene lugar a escala de poro. La eficiencia volumétrica, por otra parte, hace referencia al porcentaje del yacimiento que puede contactarse en el proceso. Esta eficiencia puede dividirse entre areal y vertical, y depende tanto de cada tecnología como de las configuraciones mecánicas que se instalen en el proceso (Green y Willhite, 1998).

Por lo tanto, dependiendo de las variables que se verán afectadas en cada una de las tecnologías emergentes, es posible inferir si su efecto es areal, vertical y/o de desplazamiento y de esta forma establecer un potencial de eficiencia de recobro.

\section{Reservas susceptibles de aplicación}

Permite estimar el potencial impacto de la tecnología en términos de incorporación de reservas. Para su cálculo se requiere en primera instancia definir las ventanas de operación o parámetros de screening técnico, los cuales son propios de cada método de recobro mejorado (Díaz, Navarro \& Tavera, 2007); ésta tarea será más compleja en la medida que la tecnología sea emergente, dado que será difícil validar los parámetros de screening, no obstante, la definición se hará con base en la revisión de literatura de casos de éxito, resultados de proyectos, pruebas en campo, o en su ausencia pruebas laboratorio.

Los parámetros definidos para el caso de las tecnologías emergentes de recobro químico son: 
viscosidad, permeabilidad, porosidad, profundidad, presión, temperatura, salinidad, saturación de aceite remanente y mecanismo de empuje del campo; evaluar entonces los rangos de aplicación de cada una de las tecnologías emergentes es el primer paso para establecer las reservas susceptibles de aplicación de nuevas tecnologías. En la tabla 2 se muestran los datos asociados a cada una de las tecnologías preseleccionadas.

Tabla 2. Ventanas de operación de las tecnologías preseleccionadas.

\begin{tabular}{|c|c|c|c|c|c|c|c|c|c|}
\hline & $\begin{array}{c}\mu \\
(\mathbf{c p})\end{array}$ & $\begin{array}{l}\text { API } \\
\left({ }^{\circ}\right)\end{array}$ & $\underset{(\mathrm{md})}{\mathrm{K}}$ & $(\%)$ & $\begin{array}{c}\text { Profundidad } \\
\text { (ft) }\end{array}$ & $\begin{array}{c}\mathbf{T} \\
\left({ }^{\circ} \mathbf{F}\right)\end{array}$ & $\begin{array}{l}\text { Salinidad } \\
\text { (ppm) }\end{array}$ & $\begin{array}{c}\text { So remanente } \\
(\%)\end{array}$ & $\begin{array}{l}\text { Sw } \\
(\%)\end{array}$ \\
\hline $\begin{array}{c}\text { Polímeros } \\
\text { modificados }\end{array}$ & $<5.400$ & No Registra & $1,8-5.500$ & $>10$ & $<9.000$ & $<248$ & $<180.000$ & $>50$ & $\begin{array}{c}\text { No } \\
\text { Registra }\end{array}$ \\
\hline $\begin{array}{c}\text { Nanotecnología } \\
\text { para EOR }\end{array}$ & $<5.400$ & No Registra & $1,8-5.500$ & $>10$ & $<9.000$ & $<248$ & $<180.000$ & $>30$ & $\begin{array}{c}\text { No } \\
\text { Registra }\end{array}$ \\
\hline $\begin{array}{c}\text { Nuevos } \\
\text { Surfactantes }\end{array}$ & No Registra & $>15$ & $1,8-5.500$ & $>10$ & $<9.000$ & $<248$ & $<50.000$ & $>30$ & $\begin{array}{l}\text { No } \\
\text { Registra }\end{array}$ \\
\hline $\begin{array}{c}\text { Agua } \\
\text { modificada }\end{array}$ & No Registra & $>15$ & $\begin{array}{c}\text { Sin } \\
\text { Restricción }\end{array}$ & $\begin{array}{c}\text { Sin } \\
\text { Restricción }\end{array}$ & $\begin{array}{c}\text { Sin } \\
\text { Restricción }\end{array}$ & $\begin{array}{c}\text { Sin } \\
\text { Restricción }\end{array}$ & $>5.000$ & $>40$ & $>25$ \\
\hline $\begin{array}{c}\text { Disociaciones } \\
\text { de sustancias } \\
\text { químicas }\end{array}$ & $\begin{array}{c}\text { Sin } \\
\text { Restricción }\end{array}$ & No Registra & $>1.000$ & No Registra & $\begin{array}{c}\text { Sin } \\
\text { Restricción }\end{array}$ & $\begin{array}{c}\text { Sin } \\
\text { Restricción }\end{array}$ & No Registra & No Registra & ALTA \\
\hline MEOR & No Registra & $>15$ & $>50$ & $>10$ & $<7.800$ & $<176$ & $<150.000$ & $>25$ & $\begin{array}{c}\text { No } \\
\text { Registra }\end{array}$ \\
\hline
\end{tabular}

Posteriormente, se deberá estimar el potencial de reservas que podría ser impactado por las tecnologías a evaluar. Para lo cual se deben comparar las propiedades de los activos de la empresa con las ventanas de operación previamente definidas. En este punto se recomienda realizar un análisis de campos análogos, que le permitirá con la evaluación de los campos más representativos conocer el potencial de incorporación de reservas una vez la tecnología haya sido validada. Para la identificación de yacimientos/campos análogos, se recomienda la metodología de la Security and Exchange Commission (SEC) (Lee, 2009), que los define como aquellos que comparten la misma formación geológica, ambiente de depositación, estructura geológica y mecanismo de empuje; finalmente, la sumatoria del OOIP (Original Oil In Place) de los análogos identificados, permitirá junto con la eficiencia de recobro, estimar el potencial de reservas o recursos recuperables.

\section{Costos asociados}

Los modelos convencionales de evaluación de tecnologías se limitan a la inversión inicial en facilidades e infraestructura, sin embargo, los costos de inversión representan solo una fracción del costo total, se deben incluir todos los costos del ciclo de vida, LCC (Life-cycle Cost), de la tecnología incluyendo inversión inicial, costos de operación y abandono. Para el caso de tecnologías de recobro químico, los costos de operación suelen ser los más representativos, por lo que se recomienda estimar un costo por barril incremental que permita una comparación de las tecnologías. Por lo tanto, para la evaluación de este parámetro, fue necesario dividir su cálculo en dos partes, la primera correspondiente al costo de la tecnología y la segunda los costos asociados a su incorporación.

Las ecuaciones 4 y 5, representan una alternativa de la estimación del costo de la tecnología, en donde I representa la inversión inicial, $\mathrm{CO}$ los costos operacionales, CA los costos de abandono, $\mathrm{R}$, las reservas susceptibles de recuperar (previamente estimadas) y CT el costo de la tecnología.

$$
\begin{gathered}
L C C_{i}=I_{i}+\sum_{t=0}^{T} C O_{i}+C A_{i} \\
C T_{i}=\frac{L C C_{i}}{\sum_{t=0}^{T} R_{i}}
\end{gathered}
$$

Teniendo en cuenta que se trata de tecnologías emergentes, se evalúa además el costo de incorporación de la tecnología, que incluye costos de adquisición de equipos e infraestructura para pruebas de laboratorio, capacitación o contratación de profesionales, membresías, licencias software, entre otros. Estos costos 
son necesarios para alcanzar la curva de aprendizaje que permita la incorporación de cada una de las tecnologías; éste costo así como su posterior ponderación, dependerá de la experiencia y capacidad de trabajo de la empresa ejecutora en procesos EOR similares, así como de sus aliados.

\section{Comercialidad}

El objetivo de la priorización es seleccionar una o varias tecnologías que pueden ser llevadas a una prueba en campo en el mediano plazo; entonces, un criterio importante es la posible disponibilidad del producto a inyectar en el yacimiento y que su producción sea estandarizada y preferiblemente a escala comercial. Por ejemplo, se considera que una tecnología es ofrecida por un proveedor único puede incrementar factores como el nivel de incertidumbre y costos, entre otros. En tal sentido, se definen los rangos de evaluación para este criterio de comercialidad resumidos en la tabla 3.
Tabla 3. Calificación cualitativa de la comercialidad.

\begin{tabular}{|c|c|c|c|}
\hline Criterio / Valoración & Alto & Medio & Bajo \\
\hline Comercialidad & $\begin{array}{c}\text { Varios } \\
\text { proveedores }\end{array}$ & $\begin{array}{c}\text { Único } \\
\text { proveedor }\end{array}$ & $\begin{array}{c}\text { No } \\
\text { comercial }\end{array}$ \\
\hline
\end{tabular}

\section{Impacto ambiental}

Para la medición de este parámetro se parte de una estimación cualitativa que comprende los diferentes impactos que se podrían presentar evaluando las siguientes perspectivas: consumo energético, uso de recursos naturales, emisiones y uso de productos amigables ambientalmente o de baja toxicidad (tabla 4). Estos criterios resultan muy importantes y toman en cuenta las potenciales dificultades que llevaría prevenir, mitigar y corregir los impactos ambientales que pudiesen ocurrir durante el desarrollo del proyecto.

Tabla 4. Descripción calificación cualitativa de los impactos ambientales.

\begin{tabular}{|c|c|c|c|}
\hline Perspectiva & Bajo & Medio & Alto \\
\hline Consumo energético & $\begin{array}{l}\text { Menor consumo energético } \\
\text { asociado a la disminución de } \\
\text { equipos procesos y/o productos }\end{array}$ & $\begin{array}{l}\text { Consumo energético asociado } \\
\text { a inyección de polímero } \\
\text { convencional }\end{array}$ & $\begin{array}{c}\text { Mayor consumo energético } \\
\text { asociado a la necesidad de equipos, } \\
\text { procesos y/o productos }\end{array}$ \\
\hline $\begin{array}{l}\text { Uso/consumo recursos } \\
\text { naturales }\end{array}$ & $\begin{array}{l}\text { Consumo nulo de agua, gas. } \\
\text { Aprovechamiento de terrenos } \\
\text { existentes, agua de reinyección, } \\
\text { reduciendo vertimientos. }\end{array}$ & $\begin{array}{l}\text { Inyección de agua fresca en bajas } \\
\text { proporciones. } \\
\text { Consumo bajo a nulo de gas } \\
\text { Vertimiento inferior a regulación }\end{array}$ & $\begin{array}{l}\text { Inyección de altas proporciones de } \\
\text { agua fresca. } \\
\text { Alto consumo de gas } \\
\text { Vertimiento cercano o superior a } \\
\text { regulación }\end{array}$ \\
\hline Emisiones & Sin emisiones atmosféricas & $\begin{array}{l}\text { Bajas emisiones, dentro del } \\
\text { marco regulatorio }\end{array}$ & $\begin{array}{c}\text { Emisiones cercanas o superiores al } \\
\text { límite regulatorio }\end{array}$ \\
\hline Toxicidad productos usados & $\begin{array}{l}\text { Productos no tóxicos. } \\
\text { Ambientalmente amigables. }\end{array}$ & $\begin{array}{l}\text { Productos tóxicos - } \\
\text { contaminantes en cantidades } \\
\text { bajas, sin afectación ambiental. }\end{array}$ & $\begin{array}{l}\text { Productos tóxicos. } \\
\text { Altamente contaminantes }\end{array}$ \\
\hline
\end{tabular}

\section{Alineación estratégica}

En este criterio se contempla la valoración del interés del sponsor en el desarrollo de la tecnología, es decir, el grado de alineación de la tecnología con el marco estratégico de la compañía y la medida de aceptación o interés que tendría en una futura aplicación en campo. Para la evaluación de este criterio se proponen tres categorías: Tecnología solicitada, la cual hace referencia a aquellos proyectos que ya cuentan con recursos y/o que haya una solicitud directa del cliente interno. Tecnología de interés, hace referencia a aquellas tecnologías que presentan interés por parte del negocio, aunque no presente una solicitud directa para su estudio se encuentra en el mapa de ruta tecnológica de la compañía y existe interés al respecto en la compañía y la última categoría es Tecnología indiferente o sin interés. (tabla 5)Esta alineación estratégica se presenta a continuación en la tabla 5.

Tabla 5. Descripción calificación cualitativa de la alineación estratégica.

\begin{tabular}{|c|c|c|c|}
\hline $\begin{array}{c}\text { Criterio / } \\
\text { Valoración }\end{array}$ & Alto & Medio & Bajo \\
\hline $\begin{array}{c}\text { Interés } \\
\text { Sponsor }\end{array}$ & $\begin{array}{c}\text { Tecnología solicitada } \\
\text { por el cliente o } \\
\text { corporativo }\end{array}$ & $\begin{array}{c}\text { Tecnología } \\
\text { de interés }\end{array}$ & $\begin{array}{c}\text { Tecnología } \\
\text { indiferente }\end{array}$ \\
\hline
\end{tabular}


En cuanto a la selección de criterios, su definición está abierta para que una compañía, tomador de decisión o evaluador, pueda incorporar nuevos criterios o eliminar aquellos que no considere relevantes. Sin embargo, se recomienda que se involucren como mínimo factores de innovación, producción, economía y comercialidad. Los factores que se proponen en este trabajo se ajustan a las necesidades de evaluación de las tecnologías emergentes de recobro químico.

En cuanto a la valoración de los parámetros, para cada uno de ellos se estableció un rango de valoraciones, esto corresponde a la matriz $\mathrm{Cjk}$, donde $\mathrm{j}$ representa cada uno de los criterios y $\mathrm{k}$ sus rangos de valoración (tabla 6). Se puede observar que los criterios que se seleccionan tienen valoraciones en diferentes rangos, unidades, e incluso se pueden combinar parámetros cuantitativos y cualitativos. Por esta razón, es necesario asignar a cada rango de valoración un puntaje numérico discretizado, el cual se muestra como un número entre paréntesis en cada rango especificado, que permita valorar los criterios en una misma escala $(5=$ Alto valor, $3=$ Valor medio, 1 = Valor bajo). El valor discretizado puede ser definido por el tomador de decisiones, el objetivo es que todos los criterios se valoren en la misma escala.

Tabla 6. Criterios de evaluación para tecnologías emergentes de recobro químico.

\begin{tabular}{|c|c|c|c|c|}
\hline Criterio (j) & Tipo & \multicolumn{3}{|c|}{ Rangos de valoración (k) } \\
\hline $\begin{array}{l}\text { Madurez de la tecnología } \\
\text { (TRL) }\end{array}$ & Cuantitativa & (5): 7 - 9 & (3): $4-5$ & (1): $1-3$ \\
\hline Eficiencia de recobro (\%) & Cuantitativa & $(5):>30$ & (3): $10-30$ & $(1):<10$ \\
\hline $\begin{array}{l}\text { Reservas disponibles } \\
\text { Oil in place (MBls) }\end{array}$ & Cuantitativa & (5): $>20.000$ & (3): $15.000-20.000$ & $(1):<15.000$ \\
\hline $\begin{array}{c}\text { Costos asociados: } \\
\text { Costo Tecnología (CT) } \\
\text { (USD/BI) }\end{array}$ & Cuantitativa & $(5):<\$ 5$ & (3): $\$ 5-\$ 20$ & $(1):>\$ 20$ \\
\hline $\begin{array}{c}\text { Costos asociados: } \\
\text { Costo incorporación (MUSD) }\end{array}$ & Cuantitativa & $(5):<\$ 1$ & (3): $\$ 1-\$ 5$ & $(1):>\$ 5$ \\
\hline Impacto ambiental & Cualitativa & (5): BAJO & (3): MEDIO & (1): ALTO \\
\hline Comercialidad & Cualitativa & $\begin{array}{l}\text { (5): Varios } \\
\text { proveedores }\end{array}$ & (3): Único proveedor & $\begin{array}{l}\text { (1): Sin } \\
\text { comercialidad }\end{array}$ \\
\hline Alineación estratégica & Cualitativa & (5): Lo solicita & (3): Interesado & $\begin{array}{l}\text { (1): Indiferente- } \\
\text { Desinteresado }\end{array}$ \\
\hline
\end{tabular}

\section{Ponderación de criterios}

Debido a la importancia individual de los criterios y su dependencia de las condiciones del estudio, su ponderación puede estar influenciada por parámetros como las características o condiciones generales de los campos objeto de estudio, el nivel de recursos de la compañía o tomador de decisión, las políticas económicas y ambientales de la compañía, la estrategia de inversión y desarrollo de la compañía y las condiciones macroeconómicas al momento de la evaluación, entre otros.

Por lo anterior, se hace necesaria la ponderación de los criterios seleccionados, es decir, asignar un mayor valor a los criterios que sean más relevantes para la priorización, para de esta forma lograr que el análisis tecnológico permita una representación realista de las preferencias del tomador de decisión y las necesidades de la compañía. En este sentido y continuando con la metodología planteada, se define el vector $\mathrm{P}_{\mathrm{i}}$, que corresponde al peso ponderado de cada criterio, la suma de los pesos debe ser igual a 1 como se muestra en la ecuación 6.

$$
\sum P j=1
$$

Para el caso de la priorización de tecnologías emergentes de recobro químico, a cada uno de los criterios se le asignó un peso que se relaciona con las necesidades de la industria petrolera colombiana; es importante que se establezca que estos valores varían dependiendo de la aplicación que se le dé a esta metodología y de cada uno de los tomadores de decisión involucrados. En la ecuación 7 se observa el vector $\mathrm{Pj}$ construido para el caso de estudio. 


$$
P j=\left[\begin{array}{c}
P_{T R L}=0.2 \\
P_{\text {Eficiencia recobro }}=0.1 \\
P_{\text {Reservas disponibles }}=0.2 \\
P_{\text {Costo Tecnologia }}=0.15 \\
P_{\text {Costo incorporación }}=0.05 \\
P_{\text {impacto ambiental }}=0.1 \\
P_{\text {Comercialidad }}=0.1 \\
P_{\text {Alineación estratédica }}=0.1
\end{array}\right]=\left[\begin{array}{c}
0.2 \\
0.1 \\
0.2 \\
0.15 \\
0.05 \\
0.1 \\
0.1 \\
0.1
\end{array}\right]
$$

\section{Valoración de las tecnologías}

La valoración de las tecnologías es una función de cada tecnología $\left(T_{i}\right)$ evaluada en la matriz de criterios $\left(C_{j k}\right)$ y sus correspondientes rangos, lo que permite obtener una matriz Sij y que se representa a partir de la ecuación 8:

$$
S_{i j}=f\left(T_{i}, C_{j k}\right)=\left[\begin{array}{llc}
S_{11} & \cdots & S_{1 j} \\
\vdots & \ddots & \vdots \\
S_{i 1} & \cdots & S_{i j}
\end{array}\right]
$$

Para el caso de aplicación y con base en los criterios evaluados en la tabla 6 , la matriz $\mathrm{S}_{\mathrm{ij}}$ se representaría como se presenta en la ecuación 9.

Es recomendable que al aplicar esta metodología, cada matriz Sij esté acompañada de una referencia o fuente que evite subjetividades en el ejercicio y brinde herramientas para justificar la evaluación. Siendo un ejercicio estratégico, el mismo será evaluado por pares y expertos de la compañía, por lo que debe tener la solidez necesaria para su función en la definición de un alcance corporativo.

$$
S_{i j}=\left[\begin{array}{cccccccc}
1-3 & 10-30 & >20,000 & \$ 5-\$ 20 \text { USD } & \$ 1-\$ 5 \text { USD } & \text { MEDIO } & \text { VARIOS PROVEEDORES } & \text { SOLICITADO } \\
4-6 & 10-30 & 15,000-20,000 & \$ 5-\$ 20 \text { USD } & <\text { <1 USD } & \text { MEDIO } & \text { VARIOS PROVEEDORES } & \text { SOLICITADO } \\
4-6 & 10-30 & 15,000-20,000 & \$ 5-\$ 20 \text { USD } & <\text { \$ USD } & \text { BAJO } & \text { VARIOS PROVEEDORES } & \text { SOLICITADO } \\
4-6 & 10-30 & <15,000 & \$ 5-\$ 20 \text { USD } & <\text { \$1 USD } & \text { MEDIO } & \text { VARIOS PROVEEDORES } & \text { INTERESADO } \\
1-3 & >30 & >20,000 & >\$ 20 \text { USD } & >\text { \$ USD } & \text { MEDIO } & \text { UNICO PROVEEDOR } & \text { INDIFERENTE } \\
7-9 & 1030 & <15,000 & \$ 5-\$ 20 \text { USD } & \$ 1-\$ 5 \text { USD } & \text { BAJO } & \text { VARIOS PROVEEDORES } & \text { INDIFERENTE }
\end{array}\right]
$$

Como se mencionó antes, las valoraciones se encuentran en diferentes rangos y unidades, por esto se define la matriz Vij (ecuación 10), como una función de Sij, que corresponde a la conversión de la valoración de cada tecnología a un conjunto de puntajes numéricos discretizados. La matriz Vij presentará las i tecnologías valoradas en cada criterio $\mathrm{j}$, de forma cuantitativa, en un mismo rango y unidad. Para el ejemplo del caso de estudio, esto se realiza con base en los datos consignados en la tabla 6 .

$$
V_{i j}=g\left(S_{i j}\right)=\left[\begin{array}{llllllll}
1 & 3 & 5 & 3 & 3 & 3 & 5 & 5 \\
5 & 3 & 3 & 3 & 5 & 3 & 5 & 5 \\
5 & 3 & 3 & 3 & 5 & 5 & 5 & 5 \\
5 & 3 & 1 & 3 & 5 & 3 & 5 & 3 \\
1 & 5 & 5 & 1 & 1 & 3 & 3 & 1 \\
3 & 3 & 1 & 3 & 3 & 5 & 5 & 1
\end{array}\right]
$$

A partir de las definiciones ya expuestas, el ranking de las tecnologías se hace con base en el producto matricial entre $V_{i j}$ y $P_{j}$, es decir, la comparación entre los criterios valorados con respecto a los pesos asignados a cada criterio. Con esto se obtiene un vector TVi que corresponde al total del puntaje valorativo para cada tecnología (Ecuaciones 11 y 12).

$$
T V_{i}=V i j * P j
$$

$$
T V_{i}=\left[\begin{array}{llllllll}
1 & 3 & 5 & 3 & 3 & 3 & 5 & 5 \\
5 & 3 & 3 & 3 & 5 & 3 & 5 & 5 \\
5 & 3 & 3 & 3 & 5 & 5 & 5 & 5 \\
5 & 3 & 1 & 3 & 5 & 3 & 5 & 3 \\
1 & 5 & 5 & 1 & 1 & 3 & 3 & 1 \\
3 & 3 & 1 & 3 & 3 & 5 & 5 & 1
\end{array}\right] *\left[\begin{array}{c}
0.2 \\
0.1 \\
0.2 \\
0.15 \\
0.05 \\
0.1 \\
0.1 \\
0.1
\end{array}\right]=\left[\begin{array}{l}
3.4 \\
3.9 \\
4.1 \\
3.3 \\
2.6 \\
2.6
\end{array}\right]
$$

De la aplicación de la metodología se obtiene una valoración numérica para cada tecnología que permite realizar un ranking y posterior priorización. Para el caso de estudio, en la tabla 7 se muestra el resultado final de la priorización, en donde la inyección de polímeros modificados (p.e. polímeros funcionalizados, Polímeros mejorados con nanopartículas, nuevos biopolímeros, etc.) e inyección de agua modificada (p.e. composición química ajustada) se perfilan como las opciones con mayores posibilidades de implementación en los campos colombianos.

Finalmente, el desarrollo de este trabajo permitió priorizar las tecnologías emergentes de recobro químico que podrían aplicarse como alternativa para el incremento del factor de recobro e incorporación de reservas en los activos colombianos. Las tecnologías evaluadas, en orden de valoración se presentan en la tabla 7, este resultado tiene un impacto estratégico en 
la compañía y en los campos colombianos, en la medida que brinda información en pro de enfocar esfuerzos e inversiones en aquellas alternativas más promisorias con base en las necesidades de la compañía y las características de sus activos.

Tabla 7. Ranking para tecnologías emergentes de recobro químico.

\begin{tabular}{|c|c|c|}
\hline Prioridad & Tecnología (Ti) & Valoración (TVi) \\
\hline $\mathbf{1}$ & Polímeros Modificados & 4.1 \\
\hline $\mathbf{2}$ & Agua modificada & 3.9 \\
\hline $\mathbf{3}$ & Nanotecnología para EOR & 3.4 \\
\hline $\mathbf{4}$ & Nuevos surfactantes & 3.3 \\
\hline $\mathbf{5}$ & MEOR & 2.8 \\
\hline $\mathbf{6}$ & $\begin{array}{c}\text { Disociaciones de sustancias } \\
\text { químicas }\end{array}$ & 2.6 \\
\hline
\end{tabular}

Desde una perspectiva técnica, el presente trabajo contribuye con la propuesta de una metodología de priorización de tecnologías práctica y sencilla que puede ser replicada en la evaluación de procesos de recobro mejorado en general. Así mismo, la identificación de ventanas de operación para las nuevas tecnologías CEOR, permitirá incorporarlas en el abanico de alternativas que se evalúan actualmente mediante herramientas corporativas de screening y analogías.

\section{Conclusiones}

- La metodología de priorización permite realizar un ranking de las tecnologías seleccionadas como objeto de estudio, para ello usa un conjunto de criterios propios de cada evaluación a realizar que deben ajustarse a las necesidades del interesado en el proceso. En este sentido, se propone un desarrollo matemático que permite otorgar un puntaje a cada tecnología para finalmente generar un listado priorizado basado en información básica de cada una de las tecnologías. La metodología desarrollada se convierte en una herramienta de fácil uso y cuyos resultados permiten enfocar los esfuerzos de investigación y desarrollo en aquellas tecnologías con mayor probabilidad de implementación.

- La selección de los criterios de valoración y posterior asignación de pesos representa el paso clave en el proceso de priorización de tecnologías. Para realizarlo de forma adecuada, es necesario caracterizar cada una de las tecnologías identificando sus fortalezas y debilidades y contrastarlas con las necesidades de los campos de interés. Adicionalmente, este proceso debe estar alineado con la visión estratégica de la empresa. Introducir sus necesidades como criterios valorativos permite realizar selecciones acordes a los futuros planes de desarrollo.

- En cuanto a las tecnologías emergentes de recobro químico para su priorización se definieron criterios económicos y técnicos, así como preferencias potenciales de los dueños de los activos. La valoración realizada utilizando la metodología propuesta arrojó como resultado que la inyección de polímeros modificados y la inyección de agua modificada resultaron ser las tecnologías con mayor potencial de implementación en los campos colombianos. El proceso propuesto puede contribuir a enfocar los esfuerzos investigativos y de desarrollo hacia las tecnologías identificadas.

- La metodología desarrollada permite personalizar el proceso de priorización dependiendo de las características o condiciones generales de los campos objeto de estudio, el nivel de recursos de la compañía o tomador de decisión, las políticas económicas y ambientales, la estrategia de inversión y desarrollo y las condiciones macroeconómicas al momento de la evaluación, entre otros aspectos; es decir, la clave del éxito de su implementación está en el conocimiento de las necesidades de los activos y la caracterización de las tecnologías potenciales.

\section{Agradecimientos}

Agradecemos a Ecopetrol S.A., especialmente al Instituto Colombiano del Petróleo (ICP) quienes en convenio con la Universidad industrial de Santander por medio del acuerdo Marco No. 5222395 permitieron el desarrollo de la investigación y la publicación de esta.

\section{Referencias Bibliográficas}

1. Albonico, P., \& Lockhart, T. P. (1993, January). Divalent ion-resistant polymer gels for hightemperature applications: syneresis inhibiting additives. In SPE International Symposium on Oilfield Chemistry. Society of Petroleum Engineers.

2. Castro-Garcia, R. H., Maya-Toro, G. A., JimenezDiaz, R., Quintero-Perez, H. I., Díaz-Guardia, V. M., Colmenares-Vargas, K. M., ... \& PérezRomero, R. A. (2016). Polymer flooding to improve volumetric sweep efficiency in waterflooding processes. CT\&F-Ciencia, Tecnología y Futuro, 6(3), 71-90. 
3. Choudry, S. A., Müller, S., Alber, U., Riedel, F., \& Landgrebe, D. (2018). A Multidimensional Assessment and Selection Methodology: Optimized Decision-making of Joining Technologies in Automobile Body Development. Procedia Manufacturing, 21, 281-288.

4. Delgadillo-Aya, C. L., Trujillo-Portillo, M. L., Palma-Bustamante, J. M., Niz-Velasquez, E., Rodriguez, C. L., \& Leon-Martinez, N. E. (2014, June). Software Tool for the Study, Analysis and Evaluation of Enhanced Oil Recovery Processes. In SPE Energy Resources Conference. Society of Petroleum Engineers.

5. Díaz, R. J., Navarro, S. F. M., \& Tavera, C. P. S. (2007). Modelo estadístico para la realización de analogías orientadas a procesos de recobro mejorado. Fuentes: El reventón energético, $5(1), 5$.

6. Elraies, K. A., Tan, I. M., Fathaddin, M. T., \& AboJabal, A. (2011). Development of a new polymeric surfactant for chemical enhanced oil recovery. Petroleum Science and Technology, 29(14), 1521-1528.

7. Gray, M., Yeung, A., Foght, J., \& Yarranton, H. W. (2008, January). Potential microbial enhanced oil recovery processes: a critical analysis. In SPE annual technical conference and exhibition. Society of Petroleum Engineers.

8. Green D. y Willhite P. (Vol 6) (1998) Enhanced Oil Recovery. Kansas, USA: Society of Petroleum Engineering. (SPE) Textbook series.

9. J. Banke, Technology Readiness Levels Demystified, NASA, 2010.

10. Jadhunandan, P. P., \& Morrow, N. R. (1995). Effect of wettability on waterflood recovery for crude-oil/brine/rock systems. SPE reservoir engineering, 10(01), 40-46.

11. Khavkin, A. (2014, January). EOR/IORNanotechnologies: Present and Future. In 21st World Petroleum Congress. World Petroleum Congress.

12. Krumrine, P. H., Lefenfeld, M., \& Romney, G. A. (2016). Alkali Metal Silicides: A New Material for Heavy-Oil Production Processes. SPE Journal, 21(01), 22-31.
13. Kudaibergenov, S. E., \& Ciferri, A. (2007). Natural and Synthetic Polyampholytes, 2: Functions and Applications. Macromolecular Rapid Communications, 28(20), 1969-1986.

14. Lee, W. J. (2009, January). Modernization of the SEC Oil and Gas Reserves Reporting Requirements. In SPE Annual Technical Conference and Exhibition. Society of Petroleum Engineers.

15. Maudgalya, S., Knapp, R. M., \& McInerney, M. (2007, January). Microbially enhanced oil recovery technologies: a review of the past, present and future. In Production and Operations Symposium. Society of Petroleum Engineers.

16. Molano, A. M. J., Navarro, S. F. M., \& Díaz, R. J. (2014). Metodología para el diseño de baches en un proceso de inyección de polímeros para recobro mejorado, considerando fenómenos de interacción roca/fluidos. Fuentes: El reventón energético, 12(2), 6.

17. Nazari Moghaddam, R., Bahramian, A., Fakhroueian, Z., Karimi, A., \& Arya, S. (2015). Comparative study of using nanoparticles for enhanced oil recovery: wettability alteration of carbonate rocks. Energy \& Fuels, 29(4), 2111-2119.

18. Ogolo, N. A., Olafuyi, O. A., \& Onyekonwu, M. O. (2012, January). Enhanced oil recovery using nanoparticles. In SPE Saudi Arabia section technical symposium and exhibition. Society of Petroleum Engineers.

19. Portwood, J. T. (1995, January). A commercial microbial enhanced oil recovery technology: evaluation of 322 projects. In SPE Production Operations Symposium. Society of Petroleum Engineers.

20. Ragab, A. M. S. (2014). Investigating the potential of nanomaterials for enhanced oil recovery: state of art. Journal of Science and Technology, $6(1)$.

21. Reyes, J. P., Trujillo, M. L., Velásquez, E. N., \& Aya, C. L. D. (2017). Metodología para la selección y priorización de yacimientos de crudo liviano candidatos a la inyección de aire: caso de aplicación. Fuentes: El reventón energético, $15(1), 41-48$. 
22. Security and Exchange Commission, «Modernization of oil and gas reporting,» SEC, Washington D.C. , 2010.

23. Seright, R. S. (2017, April). How much polymer should be injected during a polymer flood? Review of previous and current practices. In IOR 2017-19th European Symposium on Improved Oil Recovery.

24. Tang, G. Q., \& Morrow, N. R. (1997). Salinity, temperature, oil composition, and oil recovery by waterflooding. SPE Reservoir Engineering, 12(04), 269-276.

25. Tang, G. Q., \& Morrow, N. R. (1999). Influence of brine composition and fines migration on crude oil/brine/rock interactions and oil recovery. Journal of Petroleum Science and Engineering, 24(2-4), 99-111.

26. Tapias Hernandez, F. A., Lizcano Niño, J. C., Moreno, L., \& Zanoni, R. B. (2018). Effects of salts and temperature on rheological and viscoelastic behavior of low molecular weight HPAM solutions. Revista Fuentes, El Reventón Energético, 16(1).

27. Thomas, S. (2008). Enhanced oil recovery-an overview. Oil \& Gas Science and TechnologyRevue de l'IFP, 63(1), 9-19.

28. Wever, D. A. Z., Picchioni, F., \& Broekhuis, A. A. (2011). Polymers for enhanced oil recovery: a paradigm for structure-property relationship in aqueous solution. Progress in Polymer Science, 36(11), 1558-1628.

29. Wever, D. A. Z., Ramalho, G., Picchioni, F., \& Broekhuis, A. A. (2014). Acrylamide $\square \mathrm{b} \square \mathrm{N} \square$ isopropylacrylamide block copolymers: Synthesis by atomic transfer radical polymerization in water and the effect of the hydrophilic-hydrophobic ratio on the solution properties. Journal of Applied Polymer Science, 131(2).

30. Wever, D. A., Polgar, L. M., Stuart, M. C., Picchioni, F., \& Broekhuis, A. A. (2013). Polymer molecular architecture as a tool for controlling the rheological properties of aqueous polyacrylamide solutions for enhanced oil recovery. Industrial \& Engineering Chemistry Research, 52(47), 16993-17005.

31. Winnik, M. A., \& Yekta, A. (1997). Associative polymers in aqueous solution. Current opinion in colloid \& interface science, 2(4), 424-436.

32. Wu, Y., Mahmoudkhani, A., Watson, P., Fenderson, T. R., \& Nair, M. (2012, January). Development of new polymers with better performance under conditions of high temperature and high salinity. In SPE EOR Conference at Oil and Gas West Asia. Society of Petroleum Engineers.

33. Xingcai, W., \& Xiong, C. (2015). A novel particletype polymer and IOR/EOR property evalution.

Recepción: 21 de septiembre de 2018

Aceptación: 19 de noviembre de 2018 